\title{
CRANIAL POLYNEUROPATHY CAUSED BY VERTEBROBASILAR DOLICHOECTASIA
}

\author{
Tay Poh Sen ${ }^{1 *}$, Noor Aida Mat Daud ${ }^{2}$, Hilwati Hashim ${ }^{3}$ \\ ${ }^{1}$ Dept of Radiology, Hospital Kuala Lumpur, Malaysia. \\ ${ }^{2}$ Dept of Radiology, Hospital Sungai Buloh, Selangor, Malaysia. \\ ${ }^{3}$ Dept of Radiology, Faculty of Medicine, Universiti Teknologi Mara, Malaysia.
}

*Corresponding author:

Dr Tay Poh Sen, Department of Radiology, Hospital Kuala Lumpur (HKL), Malaysia. Email: pohsen@gmail.com

DOI: https://doi.org/10.32896/cvns.v2n1.1-4

Published: 07.08.2020

\begin{abstract}
Vertebrobasilar Dolichoectasia (VBD) is a condition characterized by abnormal elongation, dilatation and tortuosity of the vertebrobasilar system. It is usually asymptomatic but rarely, it can present with cranial nerve compression symptoms. We present a case of simultaneous trigeminal neuralgia and hypoglossal nerve palsy due to compression by VBD. Neuroimaging plays an important role in diagnosing this condition so that further treatment can be provided.
\end{abstract}

Keywords: Vertebrobasilar Dolichoectasia, VBD, trigeminal neuralgia, hypoglossal nerve palsy, cranial polyneuropathy.

\section{INTRODUCTION}

Neurovascular compression is one of the most important cause to be considered in patient presented with cranial neuropathy. Direct compression of cranial nerves by vertebral or basilar arteries is extremely rare and can be seen in patient with vertebrobasilar dolichoectasia (VBD). We report a rare case of vertebrobasilar dolichoectasia which causes multiple cranial nerves compression and polyneuropathy.

\section{CASE PRESENTATION}

A 50-year-old man with underlying hypertension, diabetes mellitus and dyslipidemia presented to our hospital with a 3month history of right sided jaw pain which has worsened in the past 2 days. The pain was localized to the right lower facial region with a pain score of 10/10. The pain was sharp in nature and triggered by touching or mouth opening. He had previously visited a dental clinic suspecting that he had dental caries and had his right lower $2^{\text {nd }}$ molar extracted. Despite the tooth extraction, the pain didn't subside. He was subsequently started on Carbamazepine 200mg TDS and Gabapentin $300 \mathrm{mg}$ ON which managed to control his pain since then.

On cranial nerves examination, there was no sensory abnormality at the trigeminal area, no facial weakness and cranial reflex was present. There was atrophy at the right side of the tongue with deviation to the right, signifying right hypoglossal nerve palsy. The rest of the cranial nerves and neurological examinations were normal. MRI brain was performed which showed dilated, elongated and tortuous vertebrobasilar arteries in keeping with dolichoectasia. The abnormal vertebrobasilar arteries caused compression and distortion onto multiple right-sided cranial nerves, which included trigeminal, facial, vestibulocochlear and hypoglossal nerves [Figure 1 and 2]. CTA Cerebral Artery was also performed and further confirmed the diagnosis of vertebrobasilar dolichoectasia [Figure 3].

The patient was subsequently planned for microvascular decompression (MVD) surgery by the neurosurgical team.

\section{DISCUSSION}

Vertebrobasilar Dolichoectasia (VBD) is an abnormal dilatation, elongation and tortuosity of the vertebrobasilar arteries. Dolichoectasia can occur at any of the intracranial arteries, but it is more commonly found in the vertebrobasilar arteries $^{1}$. The diagnosis of VBD can be made if the diameter of the basilar artery measures greater than $4.5 \mathrm{~mm}$ with evidence of elongation of the basilar artery i.e. basilar artery lies lateral to the clivus/dorsum sellae or it bifurcates above the plane of suprasellar cistern. ${ }^{2}$

The exact etiology for dolichoectasia is still unknown but it is postulated that severe atherosclerosis and hypertensive are likely to be responsible. However, some authors believe that congenital anomalies may also be a cause as dolichoectasia is also seen in young healthy patients and histological examination of the diseased vessels reveal defect in the internal elastic lamina and smooth muscle atrophy. ${ }^{3}$

Cranial nerves compression with neuropathy is one of the clinical presentations of VBD as shown in our case. Trigeminal and facial nerves are two of the most common cranial nerves to be involved. ${ }^{4}$ Most patients with VBD are asymptomatic, likely because cranial nerves compression in VBD is a gradual progressive process as such that the brainstem can functionally tolerate severe distortion without producing overt clinical symptoms. ${ }^{5}$ This is demonstrated in our case as multiple cranial nerves are compressed and distorted, but he presented only with trigeminal neuralgia and hypoglossal nerve palsy. 
The diagnosis of VBD can only be made with cross sectional imaging such as CT and MRI. With a patient presenting with cranial polyneuropathy, MRI with Constructive Imaging In Steady State (CISS) sequence is essential to identify any evidence of neurovascular compression. CISS sequence produce high resolution images using heavily $\mathrm{T} 2$ weighted three-dimensional gradient echo technique which provides good contrast between cerebrospinal fluid and cranial nerves. MR angiography or CT angiography will be needed to confirm the diagnosis of VBD.

In patient presented with trigeminal neuralgia (TN) from neurovascular compression, medical therapy with anticonvulsant is usually the first line treatment. Systematic review has shown that carbamazepine is the most effective drug for $\mathrm{TN}$ and should be used for initial management. Second line medication would include oxcarbazepine, gabapentin, sodium valproate, phenytoin and lamtrigine. ${ }^{6,7}$ With progression of symptoms or evidence of other cranial nerves involvement, surgical decompressive surgery i.e. microvascular decompression (MVD) or radiosurgery should be considered.

\section{CONCLUSION}

VBD is an unusual cause for trigeminal neuralgia and other cranial neuropathy. It is important for clinician to be aware of this entity. If a patient present with unusual combination of cranial nerves palsies, VBD should be considered as one of the differential diagnosis and further evaluation with MRI can be performed.

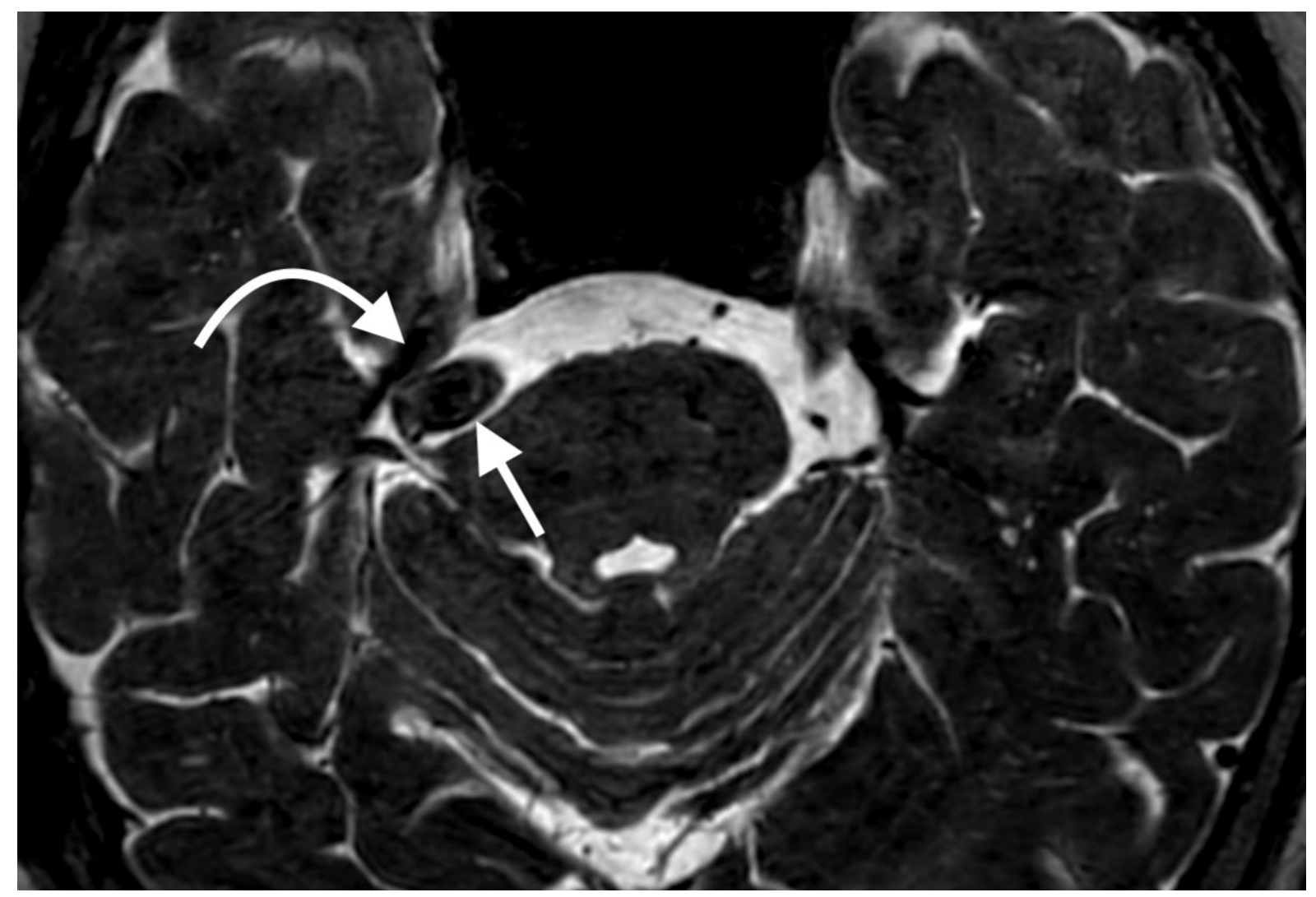

Figure 1: Axial CISS 3D sequence at the level of pons. The basilar artery is dilated and tortuous (straight arrow). It is located lateral to the clivus and compresses onto the right trigeminal nerve (curved arrow). Note the normal left trigeminal nerve. 


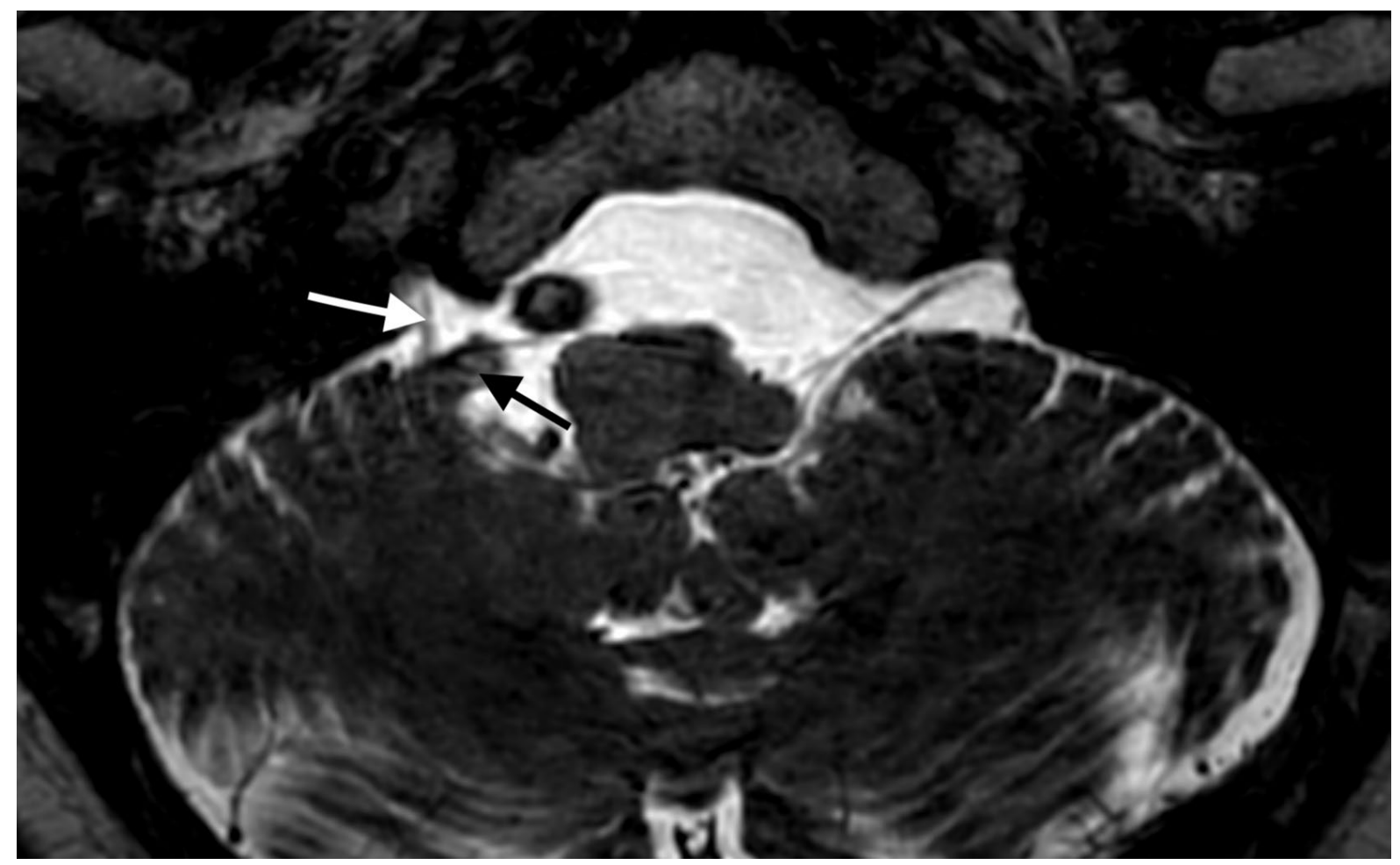

Figure 2: Axial CISS 3D sequence at the level of medulla. The dilated right vertebral artery (black arrow) compresses onto the right hypoglossal nerve (white arrow). Note the normal left hypoglossal nerve.

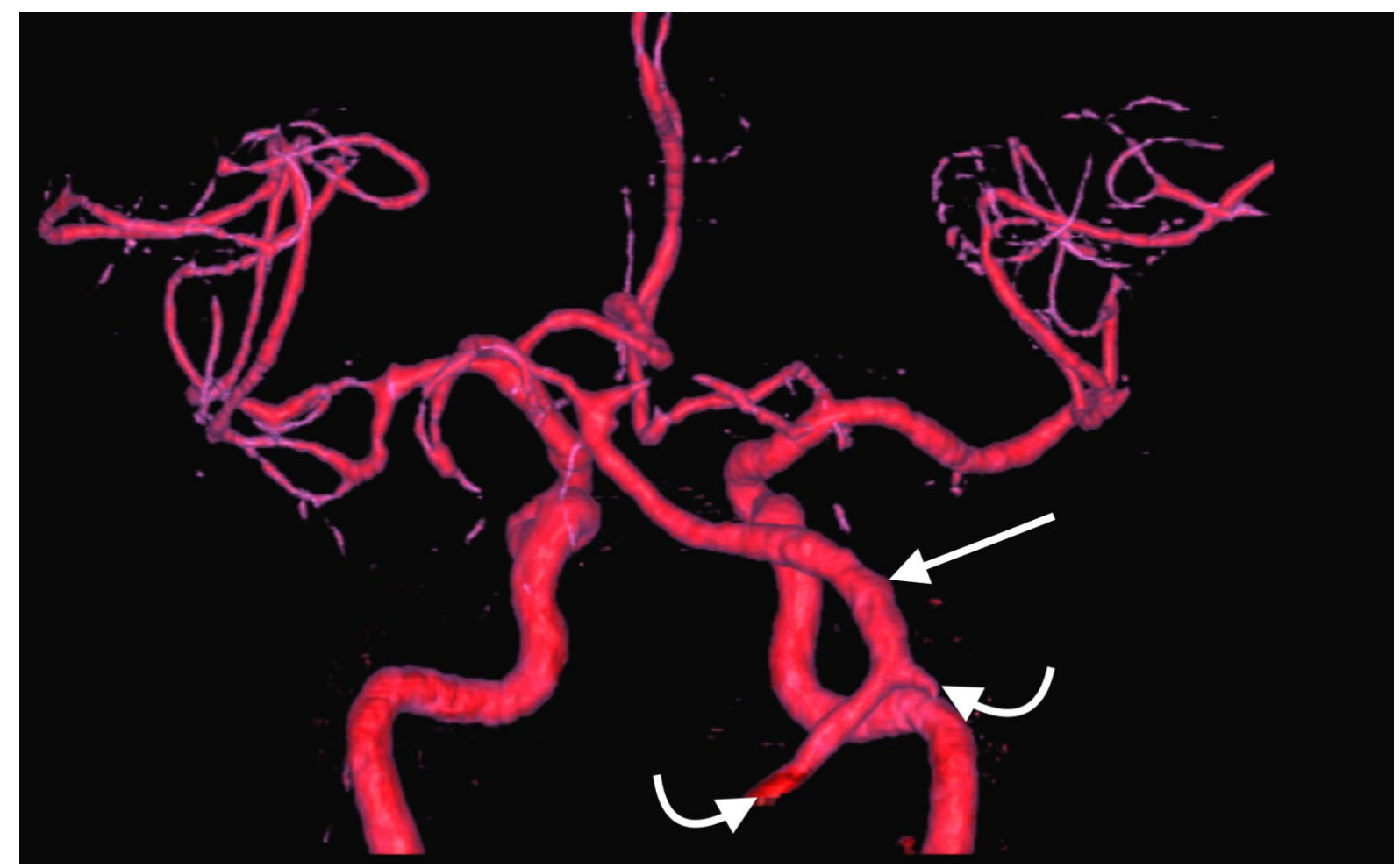

Figure 3: Volume Rendering image of the CTA shows vertebrobasilar artery dolichoectasia. Note the dilated and tortuous vertebral arteries (curved arrows) and basilar artery (straight arrow). There are irregularities along the basilar artery likely due to atherosclerotic changes. 


\section{REFERENCES}

1. Ince B, Petty GW, Brown Jr RD, Chu CP, Sicks JF and Whisnant JP. Dolichoectasia of intracranial arteries in patients with first ischemic stroke. Neurology. 1998;50(6):1694-1698

2. Love $\mathrm{S}$ and Coakham HB. Trigeminal neuralgia: pathology and pathogenesis. Brain. 2001;124(12):23472360

3. Passero SG and Rossi S. Natural history of vertebrobasilar dolichoectasia. Neurology. 2008;70(1):66-72

4. Baquero M. Vertebrobasilar dolichoectasia. Revista de Neurologia. 1998;26(149):143-148
5. De Azambuja Pereira-Filho A, de Barros Faria M, Bleil $\mathrm{C}$ and Kraemer JL. Brainstem compression syndrome caused by vertebrobasilar dolichoectasia. Arquivos de Neuro-Psiquitatria. 2008;66(2):408-411

6. Jorns TP, Zakrzewska JM. Evidence-based approach to the medical management of trigeminal neuralgia. $\mathrm{Br} \mathrm{J}$ Neurosurg. 2007;21:253-61

7. Noma N, Kobayashi A, Kamo $\mathrm{H}$ and Imamura $\mathrm{Y}$. Trigeminal neuralgia due to vertebrobasilar dolichoectasia: Three case reports. Oral Surgery Oral Medicine Oral Pathology Oral Radiology.2009;108(3):e50-55 